\title{
Patterns of hind-wing degeneration in Japanese riffle beetles (Coleoptera: Elmidae)
}

\author{
Masakazu HAYASHI ${ }^{1}$, Simon D. SONG ${ }^{2}$ and TeiJI SOTA ${ }^{2 *}$ \\ ${ }^{1}$ Hoshizaki Green Foundation, Izumo, Shimane, 691-0076, Japan; e-mail: hgf-haya@green-f.or.jp \\ ${ }^{2}$ Department of Zoology, Graduate School of Science, Kyoto University, Sakyo, Kyoto, 606-8502, Japan; \\ e-mail: sota@terra.zool.kyoto-u.ac.jp
}

Key words. Coleoptera, Elmidae, aquatic beetle, body shape, body size, dimorphism, flightlessness

\begin{abstract}
We conducted a molecular phylogenetic analysis based on mitochondrial cytochrome oxidase subunit I and nuclear $28 \mathrm{~S}$ rRNA gene sequences of species of Japanese elmids ( 23 species from 12 genera) and examined the hind-wings of 24 species in order to determine the incidence of hind-wing degeneration among species and the presence of dimorphic species with respect to hindwing degeneration. Based on the molecular phylogenetic analysis, we determined that the previously separated winged and wingless species, Stenelmis vulgaris and S. miyamotoi, and Leptelmis gracilis and L. parallela, are two forms of the same species. Of the 24 species whose hind wings were studied, we found apterous ( 3 species of Zaitzeviaria), brachypterous ( 2 species of each of Optioservus and Paramacronychus) and dimorphic species (2 species as above) in separate clades of the phylogeny. These were the smallest or medium-sized species. Dimorphic species occurred in mid- to downstream areas and used reeds and wood as substrates. The percentage of species with hind-wing degeneration (wingless or dimorphic) was high among the species (29\%) studied compared to the perceived percentage for temperate beetles $(<10 \%)$. Thus, we found that the degeneration of hind wings has occurred repeatedly in these elmid species. However, we identified only ambiguous habitat and life history correlates of hind-wing degeneration, and the adaptive significance of hind-wing degeneration in these species of elmids remains unclear.
\end{abstract}

\section{INTRODUCTION}

Hind-wing degeneration is known to occur in many insect groups, including Coleoptera (Roff, 1990). Although flight is advantageous when searching for food, mates and a suitable habitat, the production and use of the flight apparatus is costly, and degeneration of flight muscles and wings occurs based on the trade-off between flight capability and reproduction (Roff, 1990; Roff \& Fairbairn, 1991; Wagner \& Liebherr, 1992). A recent study suggested that the loss of flight ability promotes allopatric differentiation and consequently contributes to the species richness of beetles (Ikeda et al., 2012).

The loss of flight is rare in freshwater insects that have aquatic larvae but rely on dispersal by flight. Among Japanese Coleoptera whose larvae and adults can live under water, wingless species are limited to species in the family Elmidae (Kawai \& Tanida, 2005). Riffle beetles of the family Elmidae are small-sized aquatic insects that live in running water from their immature through adult stages, except for some periods of the adult stage when they fly outside the water (Brown, 1987; Elliott, 2008). Elmid beetles cannot swim and are subject to downstream drift. Therefore, even though streams are regarded as relatively permanent freshwater habitats where flightless species occur relatively frequently (Southwood, 1962), the ability to fly would be advantageous for locating more suitable habitats. Apterous species or dimorphic species with respect to hind wings are known to occur in Elmidae (Brown, 1987; Eliott, 2008), although the proportion of wingless and dimorphic species and the factors that affect the evolution of winglessness have not yet been elucidated. Among the Japanese species in this family, some are wingless and a few dimorphic (i.e., species with winged and wingless forms) (Ogata \& Nakajima, 2006; Kamite, 2009). Moreover, Inoue \& Nakajima (2009) note the possibility of two additional dimorphic species in which the winged and wingless forms are currently treated as different species (Stenelmis vulgaris and $S$. miyamotoi; and Leptelmis parallela and L. gracilis). Hayashi \& Sota (2010) also refer to this possibility based on the fact the two species in each pair are indistinguishable based on their mitochondrial COI gene sequences. These pairs of winged and wingless species differ slightly in the shapes of their bodies, which are likely to be related to the presence or absence of hind wings and flight muscles, which may represent a confusing pattern of dimorphism, as is known for species of Zaitzeviaria (Ogata \& Nakajima, 2006) and Heterlimnius (Kamite, 2009). In general, wingless species of beetles have narrower basal elytra than winged species, but this structural difference might be expected to be rare within species. In Japan, there are approximately 50 species of elmid beetles, classified into 17 genera (Satô, 1985, 1999; Jeng \& Yang, 1998; Yoshitomi \& Satô, 2005; Kamite et al., 2006; Yoshitomi \& Nakajima, 2007; Kamite, 2009). Their phylogenetic relationships and hind-wing states are not well studied.

In this study, we aimed to elucidate patterns in hindwing degeneration in Japanese elmids in relation to phy-

\footnotetext{
* Corresponding author.
} 
logeny, habitat and life history (body size). We first analyzed phylogenetic relationships among species of Japanese Elmidae to determine whether two pairs of macropterous and apterous species are in fact two species that have macropterous and apterous forms. We used a molecular phylogeny to address these taxonomic and phylogenetic questions because of the difficulties inherent in interpreting such patterns from morphological data alone. We then describe the hind-wing condition of each of the elmid species studied and discuss the occurrence of different hind-wing forms in relation to phylogeny, habitat and body size.

\section{MATERIAL AND METHODS}

\section{Sampling}

We collected adult specimens of 23 species of Elmidae in Japan for DNA extraction (Table 1). We used Satô (1985) to assist with the identification of the species. Individuals collected for DNA extraction were preserved in $99 \%$ ethanol. Adult beetles that were collected for the morphological analysis were preserved as dry specimens. Data for five species in the genus Eubrianax (Psephenidae) studied by Hayashi et al. (2012) were included as out-group taxa based on their placement as near relatives of Elmidae in Hunt et al. (2007). For the morphological analysis, we used a total of 481 specimens representing 24 species, including the 23 species from which DNA was extracted and one additional species (Dryopomorphus nakanei) [Supplementary Online Information (SOI) Tables S1-2]. All specimens used in this study are kept by M.H. at Hoshizaki Green Foundation, Izumo, Japan.

\section{DNA extraction and sequencing}

Total genomic DNA was extracted from the tissues of adults or larvae that were preserved in $99 \%$ ethanol using a DNeasy Blood \& Tissue Kit (Qiagen, Valencia, CA) or a Wizard Genomic DNA Purification Kit (Promega, Madison, WI). We used partial sequences of the mitochondrial cytochrome oxidase subunit I (COI) gene and the nuclear 28S rRNA (28S) gene for the phylogenetic analysis. The COI gene region was PCRamplified using the primers C1-J-2195 (5'-TTG ATT TTT TGG TCA TCC AGA AGT-3') and TL2-N-3014 (5'-TCC AAT GCA CTA ATC TGC CAT ATT A-3') (Simon et al., 1994). The nuclear 28S rDNA gene region was PCR-amplified using primers 28S-01 (5'-GAC TAC CCC CTG AAT TTA AGC AT-3') and 28S-R01 (5'-GAC TCC TTG GTC CGT GTT TCA AG-3') (Kim et al., 2000). Purified PCR products were used in dye terminator cycle-sequencing reactions using an ABI PRISM BigDye Terminator Cycle Sequencing FS Ready Reaction Kit, and the products were sequenced using an ABI3130XL sequencer (Applied Biosystems, Foster City, CA). Sequence data obtained in this study were deposited in DDBJ/GenBank (accession numbers AB764139-AB764228).

\section{Phylogenetic analyses}

In addition to newly obtained sequences, we used previously obtained COI gene sequences for elmid species (Hayashi \& Sota, 2010). Sequences obtained from larval specimens in the previous study were included only when the sequences were unique compared to those obtained from adult specimens. The larval specimens were identified to genus and species (whenever possible), based on morphology using a key by Hayashi \& Sota (2010) or based on monophyletic grouping with sequences from adult specimens of a single species in the COI gene tree. Note that we included sequences from larvae for thoroughness but did not use them for consideration of dimorphic or cryptic species.
The GenBank/DDBJ accession numbers for sequences for individual specimens are given in Table 1 . The alignment of COI sequences was done manually. The $28 S$ sequences were aligned using Clustal X version 1.83 (Chenna et al., 2003) with default parameter settings. The $C O I$ sequence $(814 \mathrm{bp})$ corresponded to positions 2196-3009 of the Drosophila yakuba COI sequence (Clary \& Wolstenholme, 1985), and the data set was aligned manually. Bayesian analyses and maximum-likelihood (ML) analyses were performed using MrBayes ver. 3.1.2 (Huelsenbeck \& Ronquist, 2001) and Treefinder ver. March 2011 (Jobb et al., 2004), respectively, for the COI and $28 S$ data separately and for a combined analysis using both genes. Substitution models for the Bayesian and ML analyses and the optimal partitioning schemes were selected using KAKUSAN ver. 4 (Tanabe, 2007). Different codon positions for COI were treated as different partitions, while $28 S$ was treated as a single partition. Selected models were $\mathrm{SYM}+\mathrm{G}$ for $28 S$ and $\mathrm{GTR}+\mathrm{G}$ for each COI partition. In the simultaneous analyses with two genes, these same substitution models were applied to each of the four partitions. In the Bayesian analyses with MrBayes, we ran 2 million generations of Markov chain Monte Carlo with sampling at every 200 generations and obtained 50\% majority rule consensus trees after removing the initial 1000 trees as burn-in. In the ML analyses, we performed bootstrap analyses with 100 replications in order to assess node credibility.

\section{Morphology and ecology}

We obtained data on body dimensions and ecological characters for 24 species (including D. nakanei, which was not used in the analysis of DNA). For each species, we observed the condition of the hind wings for 10-353 specimens and classified them as macropterous, brachypterous or apterous (see Table S1 for collection records). We also measured body length, maximum thoracic width and maximum elytron width for 8-45 specimens per species (see Table S2 for details). Body length was measured as the distance between the vertex of the head and the tip of an elytron in lateral view. The riverine habitat of each species was classified as headstream, upper stream, midstream or downstream. Ponds and lakes were described as lentic (L). The substrates to which larvae or adults were attached were classified as wood, gravel, sand, reed or moss. Each species was also checked for the occurrence of flight toward light as evidence of actual flight behaviour.

\section{Statistical analysis}

We conducted Fisher's exact test to determine the occurrence of different wing forms across different habitat and substrate types using R version 2.15.2 (R Foundation for Statistical Computing, Vienna). Differences in body size of the different wing forms in dimorphic species were examined using $t$-tests. An analysis of covariance was used to determine the effects of wing form and body length on thoracic and elytron widths in dimorphic species. Statistical analyses (except for Fischer's exact test) were carried out using the software JMP version 10 (SAS Institute, Carry, NC).

\section{RESULTS}

\section{Molecular phylogeny and recognition of dimorphic species}

The Bayesian inference and maximum-likelihood analysis produced similar phylogenies; Fig. 1 and SOI Figs S1-2 show the Bayesian consensus topologies. The COI gene tree (Fig. S1) has a star-like topology due to low resolution of the deep splits. There were no nucleotide differences in the COI gene sequences of Stenelmis 
TABLE 1. Specimen number, locality and DDBJ/GenBank accession numbers for their $C O I$ and $28 S$ gene sequences for species studied.

\begin{tabular}{|c|c|c|c|c|}
\hline Ind. No. & Species & Locality & $\begin{array}{c}\text { COI } \\
\text { (access. no.) }\end{array}$ & $\begin{array}{c}28 \mathrm{~S} \\
\text { (access. no.) }\end{array}$ \\
\hline$\overline{\text { HD111 }}$ & Dryopomorphus extraneus Hinton & Chihaya, Chihayaakasaka, Osaka & $\mathrm{AB} 764139$ & $\mathrm{AB} 764139$ \\
\hline HD1 12 & Dryopomorphus extraneus Hinton & R. Yoshidagawa, Sugito, Yoshida, Shimane & AB764140 & AB764140 \\
\hline HD037 & Graphelmis shirahatai (Nomura) & R. Hiikawa, Shimamura, Izumo, Shimane & GU816111 & AB764159 \\
\hline HD038 & Graphelmis shirahatai (Nomura) & R. Hiikawa, Shimamura, Izumo, Shimane & GU816112 & AB764160 \\
\hline HD039 & Graphelmis shirahatai (Nomura) & R. Hiikawa, Shimamura, Izumo, Shimane & $=\mathrm{GU} 816112$ & - \\
\hline HD055 (L) & Grouvellinus marginatus (Kono) & R. Hatagawa, Tanbe, Sada-cho, Izumo, Shimane & $=\mathrm{GU} 816130$ & - \\
\hline HD049 & Grouvellinus marginatus (Kono) & R. Hatagawa, Tanbe, Sada-cho, Izumo, Shimane & GU816127 & AB764161 \\
\hline HD050 & Grouvellinus marginatus (Kono) & R. Hiikawa, Shimamura, Izumo, Shimane & GU816128 & AB764162 \\
\hline HD051 & Grouvellinus marginatus (Kono) & R. Hiikawa, Shimamura, Izumo, Shimane & $=\mathrm{GU} 816127$ & AB764163 \\
\hline HD052 & Grouvellinus nitidus Nomura & R. Syojingawa, Ichinotani, Daisen-cho, Tottori & GU816133 & AB764164 \\
\hline HD053 & Grouvellinus nitidus Nomura & R. Syojingawa, Ichinotani, Daisen-cho, Tottori & GU816134 & AB764165 \\
\hline HD054 & Grouvellinus nitidus Nomura & R. Syojingawa, Ichinotani, Daisen-cho, Tottori & GU816135 & - \\
\hline HD040 & Leptelmis gracilis Sharp & R. Hiikawa, Shimamura, Izumo, Shimane & GU816126 & AB764166 \\
\hline HD117 & Leptelmis gracilis Sharp & R. Hiikawa, Shimamura, Izumo, Shimane & AB764142 & AB764167 \\
\hline HD1 18 & Leptelmis gracilis Sharp & R. Hiikawa, Shimamura, Izumo, Shimane & AB764143 & AB764168 \\
\hline HD119 & Leptelmis gracilis Sharp & R. Hiikawa, Shimamura, Izumo, Shimane & $=\mathrm{GU} 816126$ & $5 \quad-$ \\
\hline HD120 & Leptelmis gracilis Sharp & R. Hossyojigawa R, Yonago, Tottori & AB764141 & AB764169 \\
\hline HD126 & Leptelmis gracilis Sharp & R. Tagigawa, Taki-cho, Izumo, Shimane & $=\mathrm{AB} 764142$ & - \\
\hline HD121 & Leptelmis parallela Nomura & R. Hiikawa, Shimamura, Izumo, Shimane & $=\mathrm{AB} 764141$ & $\mathrm{AB} 764170$ \\
\hline HD122 & Leptelmis parallela Nomura & R. Hiikawa, Shimamura, Izumo, Shimane & $=\mathrm{AB} 764142$ & AB764171 \\
\hline HD123 & Leptelmis parallela Nomura & R. Hiikawa, Shimamura, Izumo, Shimane & $=\mathrm{AB} 764143$ & - \\
\hline HD124 & Leptelmis parallela Nomura & R. Hiikawa, Shimamura, Izumo, Shimane & $=\mathrm{AB} 764143$ & - \\
\hline HD110 & Neoriohelmis kurosawai Non & R. Yoshidagawa, Sugito, Yoshida, Shimane & AB764144 & AB764172 \\
\hline HD128 & Neoriohelmis kurosawai Nomura & R. Kinoe-awa, Daisen-cho, Tottori & AB764145 & AB764173 \\
\hline HD129 & Neoriohelmis kurosawai Nomura & R. Kinoe-awa, Daisen-cho, Tottori & $=\mathrm{AB} 764145$ & AB764174 \\
\hline HD130 & Neoriohelmis kurosawai Nomura & R. Kinoe-awa, Daisen-cho, Tottori & $=\mathrm{AB} 764145$ & - \\
\hline HD131 & Neoriohelmis kurosawai Nomura & R. Kinoe-awa, Daisen-cho, Tottori & $=\mathrm{AB} 764145$ & - \\
\hline HD134 & Neoriohelmis kurosawai Nomura & , Toyota, Aichi & AB764146 & 5 AB764175 \\
\hline HD135 & Neoriohelmis kurosawai Nomura & Inab & $\mathrm{AB} 7$ & AB764176 \\
\hline HD066 (L) & Optioservus sp. & Sugo, Kofu-cho, Tottori & GU816140 & - \\
\hline HD067 (L) & Optioservus sp. & Sugo, Kofu-cho, Tottori & GU816141 & - \\
\hline HD068 (L) & Optioservus sp. & ttori & GU8 & - \\
\hline HD076 & Optioservus hagai $\mathrm{\Gamma}$ & idagawa & GU8 & AB764177 \\
\hline HD077 & Optioservus hagai Nomura & R. Amidagawa, Boryo, Daisen-cho, Tottori & GU816147 & AB764178 \\
\hline HD078 & Optioservus hagai Nomura & R. Amidagawa, Boryo, Daisen-cho, Tottori & AB764148 & \\
\hline HD061 & Optioservus maculatus Nomura & Sugo, & GU816137 & AB764179 \\
\hline HD063 & Optioservus maculatus Nomura & Sugo & GU816138 & AB764180 \\
\hline HD064 & Optioservus maculatus Nomura & Sugo, Kofu-cho, Tottori & GU816139 & AB764181 \\
\hline HD069 & Optioservus nitidus Nomura & R. Syojingawa, Ichinotan & GU816151 & AB764182 \\
\hline HD070 & Optioservus nitidus Nomura & R. Syojingawa, Ichinotani, Daisen-cho, Tottori & GU816149 & - \\
\hline HD072 (L) & Optioservus nitidus Nomura & R. Syojingawa, Ichinotani, Daisen-cho, Tottori & GU816150 & - \\
\hline HD073 & Optioservus sp. & Onarubara, Kofu-cho, Tottori & GU816143 & AB764183 \\
\hline HD074 & Optioservus sp. & Onarubara, Kofu-cho, Tottori & $=\mathrm{GU} 816143$ & AB764184 \\
\hline HD075 & Optioservus sp. & Onarubara, Kofu-cho, Tottori & GU816145 & - \\
\hline HD012 & Ordobrevia foveicollis (Schonfeldt) & R. Hiikawa, Shimamura, Izumo & GU816107 & AB764185 \\
\hline HD013 & Ordobrevia foveicollis (Schonfeldt) & R. Hiikawa, Shimamura, Izumo, Shimane & $=\mathrm{GU} 816107$ & AB764186 \\
\hline HD014 (L) & Ordobrevia foveicollis (Schonfeldt) & R. Hatagawa, Tanbe, Sada-cho, Izumo, Shin & GU816109 & - \\
\hline HD015 (L) & Ordobrevia foveicollis (Schonfeldt) & R. Hatagawa, Tanbe, Sada-cho, Izumo, Shimane & $=\mathrm{GU} 816107$ & - \\
\hline HD001 & Ordobrevia gotoi Nomura & R. Hatagawa, Tanbe, Sada-cho, Izumo, Shimane & GU816105 & AB764187 \\
\hline HD002 & Ordobrevia gotoi Nomura & R. Syojingawa, Ichinotani, Daisen-cho, Tottori & $=\mathrm{GU} 816105$ & AB764188 \\
\hline HD006 (L) & Ordobrevia gotoi Nomura & R. Hatagawa, Tanbe, Sada-cho, Izumo, Shimane & $=\mathrm{GU} 816105$ & - \\
\hline HD007 (L) & Ordobrevia gotoi Nomura & R. Hatagawa, Tanbe, Sada-cho, Izumo, Shimane & $=\mathrm{GU} 816105$ & - \\
\hline HD008 (L) & Ordobrevia gotoi Nomura & R. Hatagawa, Tanbe, Sada-cho, Izumo, Shimane & $=\mathrm{GU} 816105$ & - \\
\hline HD011 (L) & Ordobrevia gotoi Nomura & Idotani, Nishihayashigi, Izumo, Shimane & $=\mathrm{GU} 816105$ & - \\
\hline HD003 & Ordobrevia maculata (Nomura) & Idotani, Nishihayashigi, Izumo, Shimane & GU816106 & AB764189 \\
\hline HD004 & Ordobrevia maculata (Nomura) & Idotani, Nishihayashigi, Izumo, Shimane & $=\mathrm{GU} 816106$ & AB764190 \\
\hline HD005 & Ordobrevia maculata (Nomura) & R. Miyamotogawa, Okutagi, Taki-cho, Izumo, Shimane & $=\mathrm{GU} 816106$ & AB764191 \\
\hline HD145 & Ordobrevia maculata (Nomura) & Ganya, Dodo, Oki Is., Shimane & $=\mathrm{GU} 816106$ & AB764192 \\
\hline HD146 & Ordobrevia maculata (Nomura) & Ganya, Dodo, Oki Is., Shimane & $=\mathrm{GU} 816106$ & $5 \quad-$ \\
\hline HD107 & Paramacronychus granulatus Nomura & Chihaya, Chihayaakasaka, Osaka & GU816169 & AB764193 \\
\hline
\end{tabular}


TABLE 1 (continued).

\begin{tabular}{|c|c|c|c|c|}
\hline Ind. No. & Species & Locality & $\begin{array}{c}\text { COI } \\
\text { (access. no.) }\end{array}$ & $\begin{array}{c}28 \mathrm{~S} \\
\text { (access. no.) }\end{array}$ \\
\hline HD108 & Paramacronychus granulatus Nomura & Chihaya, Chihayaakasaka, Osaka & GU816170 & AB764194 \\
\hline HD109 (L) & Paramacronychus granulatus Nomura & Chihaya, Chihayaakasaka, Osaka & GU816171 & - \\
\hline HD041 & Pseudamophilus japonicus Nomura & R. Hiikawa, Yokota, Okuziumo, Shimane & GU816110 & AB764195 \\
\hline HD042 & Pseudamophilus japonicus Nomura & R. Hiikawa, Yokota, Okuziumo, Shimane & $=\mathrm{GU} 816110$ & AB764196 \\
\hline HD043 & Pseudamophilus japonicus Nomura & R. Hiikawa, Yokota, Okuziumo, Shimane & $=\mathrm{GU} 816110$ & - \\
\hline HD026 (L) & Stenelmis sp. & R. Hiikawa, Izumo, Shimane & GU816121 & - \\
\hline HD027 (L) & Stenelmis sp. & Hiikawa, Hinobori, Kisuki, Unnan, Shimane & GU816122 & - \\
\hline HD017 & Stenelmis miyamotoi Nomura et Nakane & R. Hiikawa, Shimamura, Izumo, Shimane & GU816113 & AB764197 \\
\hline HD018 & Stenelmis miyamotoi Nomura et Nakane & R. Hiikawa, Shimamura, Izumo, Shimane & GU816114 & AB764198 \\
\hline HD019 & Stenelmis miyamotoi Nomura et Nakane & R. Hiikawa, Shimamura, Izumo, Shimane & GU816115 & - \\
\hline HD020 & Stenelmis miyamotoi Nomura et Nakane & R. Hiikawa, Shimamura, Izumo, Shimane & $=\mathrm{GU} 816114$ & - \\
\hline HD137 & Stenelmis miyamotoi Nomura et Nakane & R. Hossyojigawa R, Yonago, Tottori & AB764149 & AB764199 \\
\hline HD138 & Stenelmis miyamotoi Nomura et Nakane & R. Hossyojigawa R, Yonago, Tottori & $=\mathrm{GU} 816113$ & AB764200 \\
\hline HD139 & Stenelmis miyamotoi Nomura et Nakane & R. Hossyojigawa R, Yonago, Tottori & $\mathrm{AB} 764150$ & - \\
\hline HD148 & Stenelmis miyamotoi Nomura et Nakane & R. Mukogawa, Sumada, Sanda, Hyogo & AB764151 & AB764201 \\
\hline HD149 & Stenelmis miyamotoi Nomura et Nakane & R. Mukogawa, Sumada, Sanda, Hyogo & $=\mathrm{GU} 816113$ & AB764202 \\
\hline HD150 & Stenelmis miyamotoi Nomura et Nakane & R. Mukogawa, Sumada, Sanda, Hyogo & $=\mathrm{GU} 816113$ & - \\
\hline HD151 & Stenelmis miyamotoi Nomura et Nakane & R. Mukogawa, Sumada, Sanda, Hyogo & $=\mathrm{GU} 816113$ & - \\
\hline HD152 & Stenelmis miyamotoi Nomura et Nakane & R. Mukogawa, Sumada, Sanda, Hyogo & $=\mathrm{GU} 816113$ & - \\
\hline HD016 (L) & Stenelmis nipponica Nomura & R. Hatagawa, Tanbe, Sada-cho, Izumo, Shimane & GU816125 & - \\
\hline HD028 & Stenelmis nipponica Nomura & Shakunouchi, Kisuki, Unnan, Shimane & GU816123 & AB764203 \\
\hline HD029 & Stenelmis nipponica Nomura & Shakunouchi, Kisuki, Unnan, Shimane & GU816124 & AB764204 \\
\hline HD021 & Stenelmis vulgaris Nomura & R. Hiikawa, Shimamura, Izumo, Shimane & $=\mathrm{GU} 816113$ & AB764205 \\
\hline HD022 & Stenelmis vulgaris Nomura & Hiikawa, Shimamura, Izumo, Shimane & GU816118 & AB764206 \\
\hline HD023 & Stenelmis vulgaris Nomura & Hiikawa, Shimamura, Izumo, Shimane & GU816119 & - \\
\hline HD136 & Stenelmis vulgaris Nomura & R. Hossyojigawa R, Yonago, Tottori & AB764152 & AB764207 \\
\hline HD147 & Stenelmis vulgaris Nomura & R. Mukogawa, Sumada, Sanda, Hyogo & AB764153 & AB764208 \\
\hline HD101 (L) & Zaitzevia sp. & R. Hiikawa, Hinobori, Kisuki, Unnan, Shimane & GU816165 & - \\
\hline HD102 (1 & Zaitzevia sp. & R. Hiikawa, Hinobori, Kisuki, Unnan, Shimane & GU816166 & - \\
\hline HD103 & Zaitzevia sp. & R. Hiikawa, Hinobori, Kisuki, Unnan, Shimane & AB764154 & - \\
\hline HD096 & Zaitzevia awana (Kono) & R. Hiikawa, Hinobori, Kisuki, Unnan, Shimane & GU816163 & AB764209 \\
\hline HD097 & Zaitzevia awana (Kono) & R. Hiikawa, Hinobori, Kisuki, Unnan, Shimane & GU816164 & AB764210 \\
\hline HD098 & Zaitzevia awana (Kono) & R. Hiikawa, Hinobori, Kisuki, Unnan, Shimane & $=\mathrm{GU} 816164$ & - \\
\hline HD099 & Zaitzevia nitida Nomura & R. Kasetsugawa, Kotoura-cho, Tottori & $=\mathrm{GU} 816164$ & AB764211 \\
\hline HD100 & Zaitzevia nitida Nomura & R. Kasetsugawa, Kotoura-cho, Tottori & GU816168 & AB764212 \\
\hline HD093 & Zaitzevia rivalis Nomura & Gakuenji, Izumo, Shimane & GU816161 & AB764213 \\
\hline HD094 & Zaitzevia rivalis Nomura & , Izumo, Shimane & $=\mathrm{GU} 816161$ & AB764214 \\
\hline HD095 & Zaitzevia rivalis Nomura & Gakuenji, Izumo, Shimane & GU816162 & AB764215 \\
\hline HD079 & Zaitzeviaria brevis (Nomura) & R. Syojingawa, Ichinotani, Daisen-cho, Tottori & GU816151 & AB764216 \\
\hline HD080 & Zaitzeviaria brevis (Nomura) & R. Hiikawa, Shimamura, Izumo, Shimane & GU816152 & AB764217 \\
\hline HD081 & Zaitzeviaria brevis (Nomura) & R. Hiikawa, Shimamura, Izumo, Shimane & GU816153 & $\mathrm{AB} 764218$ \\
\hline HD090 & Zaitzeviaria gotoi (Nomura) & Gakuenji, Izumo, Shimane & GU816160 & AB764219 \\
\hline HD091 & Zaitzeviaria gotoi (Nomura) & Gakuenji, Izumo, Shimane & $=\mathrm{GU} 816160$ & AB764220 \\
\hline HD140 & Zaitzeviaria gotoi (Nomura) & R. Choshi-gawa, Dogo, Oki Is., Shimane & $\mathrm{AB} 764155$ & AB764221 \\
\hline HD141 & Zaitzeviaria gotoi (Nomura) & R. Choshi-gawa, Dogo, Oki Is., Shimane & $=\mathrm{AB} 764155$ & AB764222 \\
\hline HD142 & Zaitzeviaria gotoi (Nomura) & R. Choshi-gawa, Dogo, Oki Is., Shimane & $=\mathrm{AB} 764155$ & - \\
\hline HD082 & Zaitzeviaria ovata (Nomura) & Idotani, Nishihayashigi, Izumo, Shimane & GU816154 & AB764223 \\
\hline HD083 & Zaitzeviaria ovata (Nomura) & Idotani, Nishihayashigi, Izumo, Shimane & GU816155 & AB764224 \\
\hline HD084 & Zaitzeviaria ovata (Nomura) & Idotani, Nishihayashigi, Izumo, Shimane & GU816156 & - \\
\hline HD085 & Zaitzeviaria ovata (Nomura) & R. Miyamagawa, Manda-cho, Izumo, Shimane & $=\mathrm{GU} 816156$ & AB764225 \\
\hline HD086 & Zaitzeviaria ovata (Nomura) & R. Miyamagawa, Manda-cho, Izumo, Shimane & $=\mathrm{GU} 816156$ & AB764226 \\
\hline HD143 & Zaitzeviaria ovata (Nomura) & Nibu, Chibu-mura, Oki Is., Shimane & $\mathrm{AB} 764156$ & AB764227 \\
\hline HD144 & Zaitzeviaria ovata (Nomura) & Nibu, Chibu-mura, Oki Is., Shimane & $=\mathrm{AB} 764156$ & AB764228 \\
\hline \multicolumn{5}{|l|}{ Outgroup } \\
\hline DR010 & Eubrianax ramicornis Kiesenwetter & Kisuki, Unnan, Shimane & EU287832 & AB675827 \\
\hline DR025 & Eubrianax granicollis Lewis & Kisuki, Unnan, Shimane & EU287819 & AB675797 \\
\hline DR093 & Eubrianax amamiensis Sato & Kinsakubaru, Amami-oshima I., Kagoshima & AB675746 & AB675792 \\
\hline DR105 & Eubrianax pellucidus Lewis & Takatani, Tsuruoka, Yamagata & AB675744 & AB675790 \\
\hline DR141 & Eubrianax loochooensis Nakane & Nago, Okinawa I. & AB675763 & AB675812 \\
\hline
\end{tabular}

L - larval specimen; the others are adults. For Elmidae, DDBJ/GenBank accession numbers followed by GU and AB were published by Hayashi \& Sota (2010) and the present study, respectively. For Eubrianax (outgroup), the accession numbers followed by EU and AB were published by Hayashi \& Sota (2008) and Hayashi et al. (2012), respectively. 


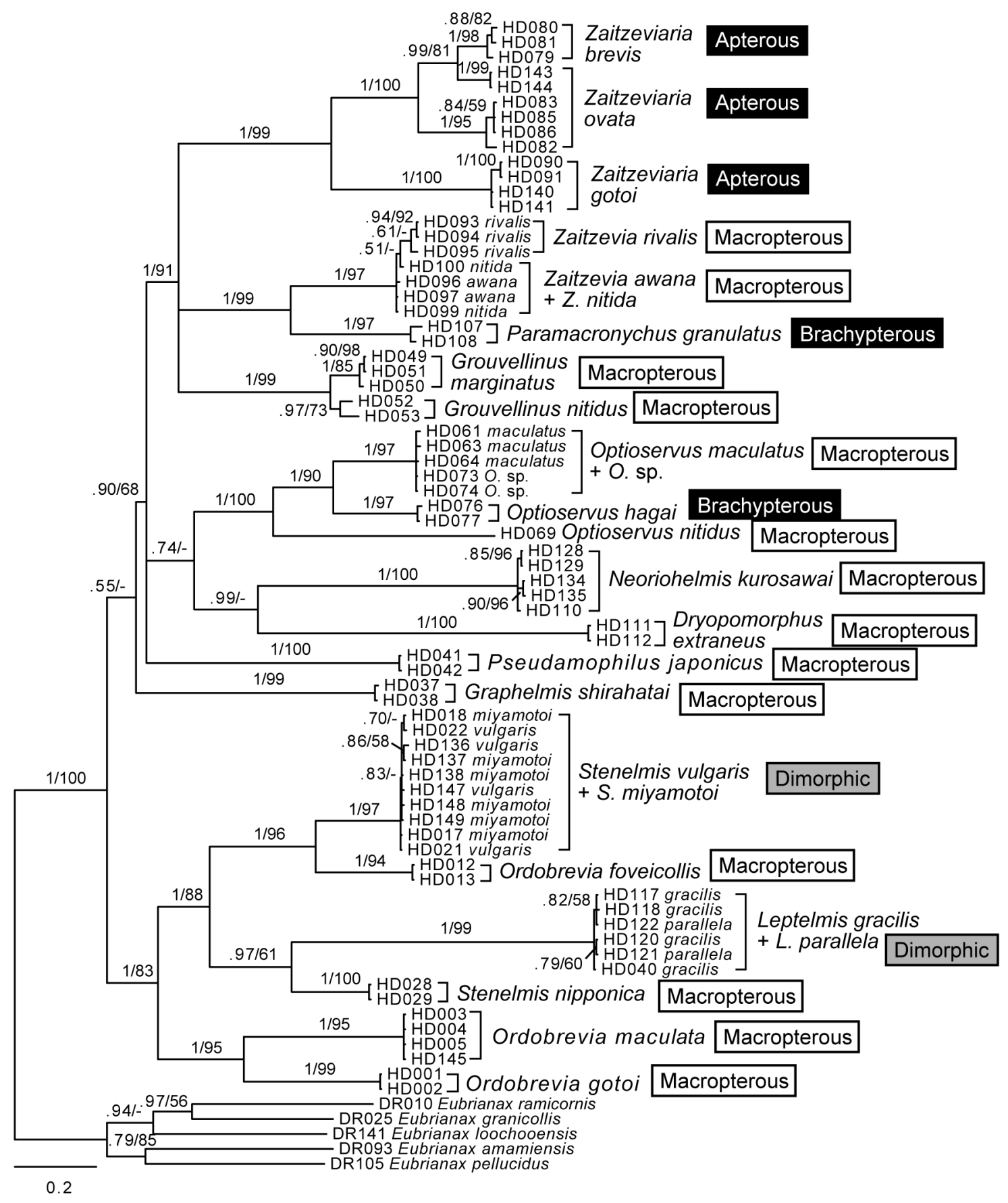

Fig. 1. The 50\% majority rule consensus tree that resulted from the Bayesian phylogenetic analysis of the combined COI and $28 S$ gene sequences. Nodal support is shown in the form of Bayesian posterior probabilities and maximum-likelihood bootstrap percentages ("-" indicates below 50\%).

miyamotoi and S. vulgaris, Leptelmis gracilis and L. parallela, Optioservus maculatus and Optioservus sp. or Zaitzevia awana and Z. nitida. In the $28 S$ gene tree (Fig. S2), species were also not distinguished within these four pairs. Thus, the results based on mitochondrial and nuclear genes were congruent. In addition, Zaitzevia rivalis shared the same $28 S$ sequence with $Z$. awana and $Z$. nitida. Furthermore, four species were paraphyletic within the $28 S$ tree (Zeitzeviaria ovata, Grouvellinus marginatus, Ordobrevia foveicollis and O. maculata). In the simultaneous analysis tree (Fig. 1), three species pairs, $S$. miyamotoi and S. vulgaris, L. gracilis and L. parallela, and O. maculatus and Optioservus sp., were monophyletic, and Z. awana and Z. nitida together formed a basal part of a paraphyletic group. At the genus level, polyphyly of Stenelmis and Ordobrevia was strongly supported. These genera together with Leptelmis formed a monophyletic group.

Of the four pairs of species that were indistinguishable using molecular markers, Stenelmis vulgaris and S. miyamotoi, and Leptelmis gracilis and L. parallela were also indistinguishable in terms of their genital morphology (Fig. 2). We hereafter treat each pair of these species as two forms of the same species (i.e., dimorphic species). 
(a)

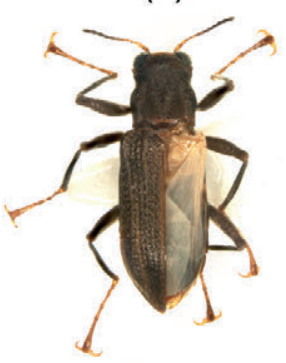

(e)

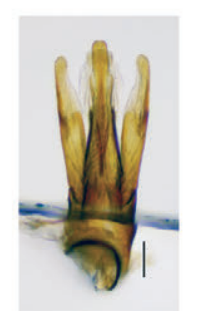

(b)

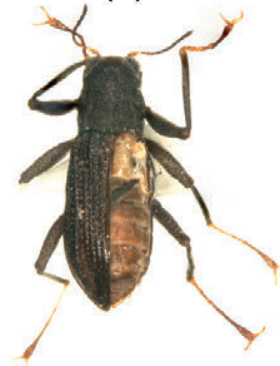

(f)

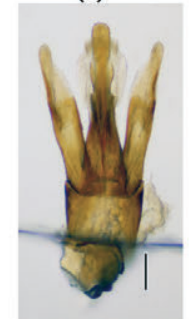

(c)

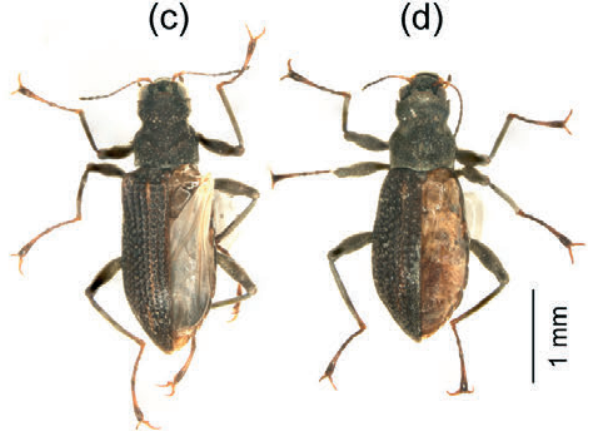

(g)

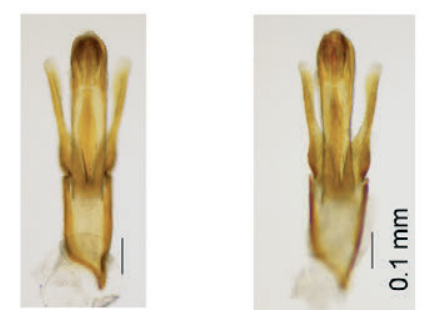

Fig. 2. Adult beetles of (a) Stenelmis vulgaris, (b) S. miyamotoi, (c) Leptelmis parallela and (d) L. gracilis. Right elytra are removed to show the condition of the hind wings. Male genitalia of (e) Stenelmis vulgaris, (f) S. miyamotoi, (g) Leptelmis parallela and (h) L. gracilis.

TABLE 2. The percentage of macropterous individuals recorded, wing type, habitat, substrate of microhabitat and tendency of each species to fly to light.

\begin{tabular}{|c|c|c|c|c|c|c|c|c|}
\hline Species & $\begin{array}{l}\% \text { macro- } \\
\text { pterous }(n)\end{array}$ & $\begin{array}{l}\text { Wing } \\
\text { type }\end{array}$ & Habitat & $\begin{array}{l}\text { Sub- } \\
\text { strate }\end{array}$ & $\begin{array}{c}\text { Tendency } \\
\text { to fly to } \\
\text { light } \\
\end{array}$ & $\begin{array}{l}\text { Body length, mm } \\
(\text { mean } \pm \text { SD })\end{array}$ & $\begin{array}{c}\text { Thoracic width, } \\
\text { mm } \\
(\text { mean } \pm \mathrm{SD})\end{array}$ & $\begin{array}{c}\text { Elytron width, } \\
\text { mm } \\
(\text { mean } \pm \mathrm{SD})\end{array}$ \\
\hline Dryopomorphus extraneus & $0.0(15)$ & $\mathrm{M}$ & $\mathrm{H}$ & $\mathrm{W}$ & - & 22) & $1.78 \pm 0.07(22)$ & $1.97 \pm 0.07(22)$ \\
\hline Dryopomorphus 1 & $00.0(10)$ & M & HUMD & $\mathrm{W}$ & - & 10) & $1.37 \pm 0.10(10)$ & $1.58 \pm 0.13(10)$ \\
\hline Stenelmis nipponica & $0.0(17)$ & M & UMD & $\mathrm{G}$ & +++ & & $0.92 \pm 0.04(20)$ & $1.17 \pm 0.04(20)$ \\
\hline Stenelmis vulgaris/ & $38.0(353)$ & D & MD & $\mathrm{R}(\mathrm{W})$ & +++ & & & \\
\hline Macropterous & & & & & & 1) & $0.70 \pm 0$ & $0.97 \pm 0$ \\
\hline Apte & & & & & & & 0.73 & $0.98 \pm$ \\
\hline Ordobre & $100.0(27)$ & M & HUM & G & - & & $0.54 \pm$ & $0.62 \pm 0.04(17)$ \\
\hline Ordobr & $100.0(20)$ & M & $\mathrm{HU}$ & G & - & & $0.65 \pm 0.05(18)$ & $0.82 \pm 0.04(18)$ \\
\hline Ordobrev & $100.0(15)$ & M & MD & $\mathrm{S}(\mathrm{W})$ & +++ & & $0.87 \pm 0.05(20)$ & $1.16 \pm 0.06(20)$ \\
\hline Leptelmi & $24.2(62)$ & $\mathrm{D}$ & $\mathrm{MD} / \mathrm{L}$ & $\mathrm{R}(\mathrm{W})$ & + & & & \\
\hline allela) & & & & & & & 0.6 & $1.00 \pm$ \\
\hline $\mathrm{Ap}$ & & & & & & & & 1.0 \\
\hline Graphe & & M & MD & b & + & & 0.87 & $1.20 \pm 0$ \\
\hline Neor & 10 & M & $\mathrm{U}$ & $\mathrm{G}$ & - & & 1.4 & $1.69 \pm 0$ \\
\hline Optio & & $\mathrm{B}$ & HUM & $\mathrm{S}$ & - & & $1.11 \pm 0$ & $1.27 \pm 0$ \\
\hline Optio & & M & & G & - & & (20) & $1.28 \pm$ \\
\hline Optio & ? & M & $\mathrm{HU}$ & $\mathrm{S}$ & - & & 0.9 & 1.13 \\
\hline Optio & 2) & M & UMD & $\mathrm{S}$ & - & $0)$ & 0.6 & $0.79 \pm$ \\
\hline Pseudamophilus japonicu. & & M & UMD & $\mathrm{W}(\mathrm{R})$ & + & & $1.63 \pm 0$ & $2.06 \pm 0.07(20)$ \\
\hline Grouvellinus & & M & & & +++ & & $0.71 \pm 0$ & $0.94 \pm 0$ \\
\hline Grou & 100.0 & M & UM & (M) & - & 1) & 0.76 & $1.00 \pm 0$ \\
\hline Paramacronychus granulatus & $5.0(20)$ & $\mathrm{B}$ & $\mathrm{HU}$ & $\mathrm{G}$ & - & $2.32 \pm 0.06(19)$ & $0.90 \pm 0.03(20)$ & $1.12 \pm 0.04(20)$ \\
\hline Zaitze & $2.4(41)$ & A & HUMD & G & - & $1.22=$ & $0.43 \pm 0.02(20)$ & $0.52 \pm 0.03(20)$ \\
\hline Zaitzeviaria ovata & $0.4(249)$ & A & HU & S & - & & $0.47 \pm 0$ & $0.60 \pm 0.03(20)$ \\
\hline Zaitzeviaria brevis & $3.4(29)$ & A & MD & $\mathrm{S}$ & - & $1.26 \pm 0.07(20)$ & $0.45 \pm 0.03(20)$ & $0.56 \pm 0.04(20)$ \\
\hline Zaitzevia awana & $100.0(85)$ & M & MD & G & +++ & $1.82 \pm 0.08(20)$ & $0.60 \pm 0.03(20)$ & $0.78 \pm 0.05(20)$ \\
\hline Zaitze & $100.0(14)$ & M & UM & $\mathrm{C}$ & - & $1.96 \pm 0.09(10)$ & $0.63 \pm 0.03(10)$ & $0.79 \pm 0.04(10)$ \\
\hline Zaitzevia rivalis & $100.0(38)$ & M & $\mathrm{HU}$ & G & - & $1.99 \pm 0.06(15)$ & $0.67 \pm 0.020(20)$ & $0.88 \pm 0.04$ \\
\hline
\end{tabular}

Wing type: M - macropterous; B - brachypterous; A - apterous; D - dimorphic. Habitat: H - head-stream; U - upper-stream; M mid-stream; D - down-stream; L - lentic. Substrate: W - wood; G - gravel; S - sand; R - reed; M - moss. Parenthesized substrates are occasionally used. Tendency to fly to light: +++, many records; +, rare; -, not known. 


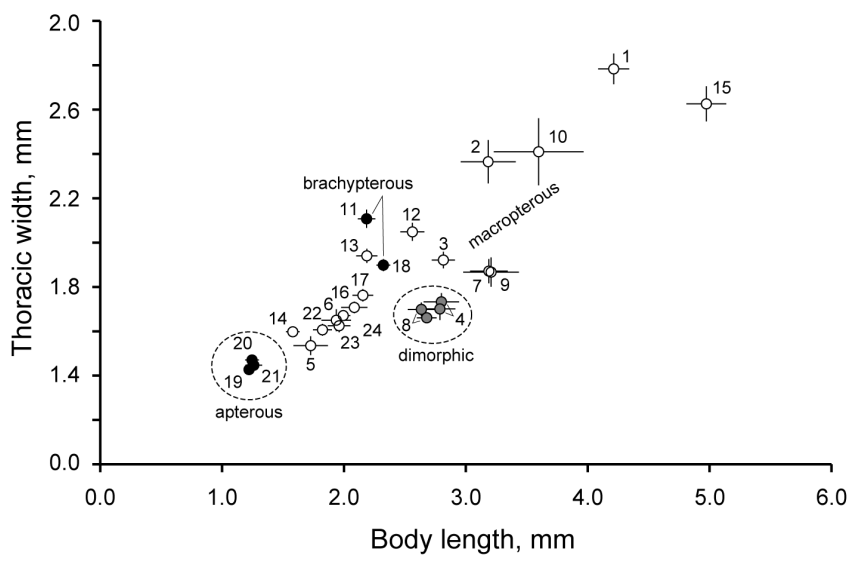

Fig. 3. Relationship between mean body length and mean thoracic width. Bars represent the standard deviation (SD). Numerals indicate species number as follows: 1 -Dryopomorphus extraneus; 2 - Dryopomorphus nakanei; 3 -Stenelmis nipponica; 4 - Stenelmis vulgaris/ S. miyamotoi; 5 -Ordobrevia gotoi; 6 - Ordobrevia maculata 7 - Ordobrevia foveicollis; 8 Leptelmis gracilis/ L. parallela; 9 - Graphelmis shirahatai; 10 Neoriohelmis kurosawai; 11 - Optioservus hagai; 12 - Optioservus sp.; 13 - Optioservus maculatus; 14 - Optioservus nitidus; 15 - Pseudamophilus japonicus; 16 - Grouvellinus marginatus; 17 - Grouvellinus nitidus; 18 - Paramacronychus granulatus; 19 - Zaitzeviaria gotoi; 20 - Zaitzeviaria ovata; 21 - Zaitzeviaria brevis; 22 - Zaitzevia awana; 23 - Zaitzevia nitida; 24 - Zaitzevia rivalis. Black circles indicate apterous or brachypterous species; grey circles, dimorphic species; open circles, macropterous species.

The other species pairs, Optioservus maculatus and Optioservus sp., and Z. awana and Z. nitida can be distinguished based on their external and genital morphology (SOI Figs S3-4; see also Hayashi, 2011; Kamite, 2011).

\section{Hind wings}

Among the 24 species studied, macroptery was common, occurring in 17 species of 9 genera ( $71 \%$ of 24 species; Table 2). Of the remaining 7 species, 2 belonging to 2 genera were brachypterous, 3 species from a single genus were apterous, and 2 species, $S$. vulgaris and $L$. gracilis, as mentioned above, had dimorphic hind wings (Table 2). Note that all of the brachypterous/apterous species sampled included at least one macropterous individual. Both macropterous and brachypterous/apterous species occurred in all the river habitats, while the two dimorphic species occurred in lower stream or still water habitats (Table 2). However, the occurrence of macropterous, dimorphic and brachypterous/apterous species did not differ statistically among the five habitats (Fisher's exact test, $P=0.3209$ ). The five substrates used by larvae and adults were used differently by macropterous, dimorphic and brachypterous/apterous species (Table 2; Fisher's exact test, $P=0.0472 ; P=0.0109$ when occasionally used substrates were not considered). Macropterous species used all substrate types, whereas dimorphic species mostly used reeds and brachypterous/apterous species gravel and sand. Flying to light was observed in $35 \%$ of the macropterous species (Table 2).
Among the species studied, macropterous species showed a wide range of body sizes, whereas the three apterous species, all species of Zaitzeviaria, were the smallest (Fig. 3). Two brachypterous species and two dimorphic species were medium-sized. Among the dimorphic species, there were no significant differences in body lengths ( $\log _{10}$-transformed) of macropterous and apterous individuals ( $t$-test: $L$. gracilis, $\mathrm{df}=1,24, t=1.1, P=$ $0.2824 ; S$. vulgaris, df $=1,43, t=-0.272, P=0.2824)$. An analysis of covariance (ANCOVA) showed that in $L$. gracilis, macropterous individuals had wider thoraces and elytron widths than apterous individuals $\left(\log _{10}\right.$-thoracic width: wing form effect, $F_{1,23}=26.8, P<0.0001 ; \log _{10^{-}}$ body length effect: $F_{1,23}=51.4, P<0.0001$; $\log 10$-elytron width: wing form effect, $F_{1,23}=12.7, P=0.0017 ; \log 10$ body length effect: $\left.F_{1,23}=26.8, P<0.0001\right)$. However, in $S$. vulgaris, the interaction effect between body length and wing form was significant, although macropterous individuals had wider thoraces on average (ANCOVA, $\log _{10}$-thoracic width: wing form effect, $F_{1,41}=23.1, P<$ 0.0001 ; $\log _{10}$-body length effect: $F_{1,41}=179.9, P<$ 0.0001 ; interaction effect, $F_{1,41}=11.2, P=0.0018$; $\log _{10^{-}}$ elytron width: wing form effect, $F_{1,41}=0.345, P=0.5602$; $\log _{10}$-body length effect: $F_{1,41}=99.3, P<0.0001$; interaction effect, $F_{1,41}=7.2, P=0.0107$ ).

\section{DISCUSSION}

The simultaneous analysis of mitochondrial and nuclear gene sequences revealed the monophyly of 9 of the 12 genera studied (Fig. 1). The remaining three genera, Stenelmis, Leptelmis and Ordobrevia were mixed in one clade in the molecular phylogeny, indicating that the classification of these genera should be reassessed. At the species level, our results suggest that Stenelmis miyamotoi Nomura \& Nakane (1958) is a synonym of $S$. vulgaris Nomura (1958) and Leptelmis parallela Nomura (1962) a synonym of $L$. gracilis Sharp (1888). The formal synonymization of these species will be made elsewhere since this study is not intended to be a taxonomic revision. Here, we discuss the pattern of hind-wing degeneration based on our recognition of these two dimorphic species.

For the 24 species in this study, apterous species were found only in Zeitzeviaria, but brachypterous and dimorphic species occurred in four different genera in different clades of the molecular phylogeny (Fig. 1). Thus, hind wing degeneration has occurred repeatedly in these Japanese elmids (Fig. 1). Note that the apterous and brachypterous species reported here contained very few $(<5 \%)$ winged individuals, suggesting that alleles for macroptery are maintained in the populations. In holometabolous insects with one-locus two-allele system for wing dimorphism, macroptery is recessive to aptery/brachyptery (Roff \& Fairbairn, 2007). Of the 25 species of Japanese elmids that were not included in this study, five, Orientelmis parvula, Heterlimnius ater, Sinonychus satoi, S. tsujunensis and Zaitzeviaria kuriharai, are apterous (Shepard, 1998; H. Yoshitomi \& Y. Kamite, pers. com.), and one, Heterlimnius hasegawai, is dimorphic (Kamite, 
2009, 2012). Thus, among all the species of Japanese elmids, the percentage of wingless species (apterous and brachypterous) is $20 \%$, and it becomes $27 \%$ when dimorphic species are included. These percentages are relatively high among coleopteran families (Roff, 1990; $<10 \%$ in temperate beetles). The loss of the ability to fly appears paradoxical for this water beetle family because they live in habitats with running water, where they are at risk of being washed away. Elmid beetles frequently drift downstream (Elliott, 2008), and therefore, the ability to fly is important for recolonizing upper stream habitats. Only L. gracilis, with hind-wing dimorphism, lives in still water in addition to running water, and only the apterous form is found in still water habitats (Satô, 1985; Yoshitomi \& Satô, 2005). Thus, flightlessness can be favoured in stable habitats, but this cannot explain the occurrence of flightless forms in running water habitats.

Flightlessness may evolve in favor of a larger reproductive capacity, which is often associated with larger body sizes. However, among the species studied, apterous species were the smallest species, and brachypterous and dimorphic species were medium-sized. Among the dimorphic species, no difference in body length was observed between macropterous and apterous forms. Thus, a clear trade-off may not exist between flight capability and reproductive capacity (i.e., body size) in elmid beetles, although a more precise measurement of reproductive capacity (e.g., number of eggs) is needed. Our results suggest that wing dimorphism is associated with the use of reeds as substrates. The use of habitats with reeds may involve factors that promote the evolution of wingless forms. However, we need to study more species in order to critically assess this possibility.

In dimorphic species of elmids, wingless species have sloping shoulders (front part of the elytra) due to degeneration of the hind wings, whereas winged species have square shoulders (Fig. 2). Wing dimorphism accompanied by dimorphism in body shape may be rare, although such an association between elytral shape and hind-wing state is recorded as an inter-specific difference. Dimorphism in body shape, as in S. vulgaris and L. gracilis, is documented for two Zeitzeviaria species (Ogata \& Nakajima, 2006) and H. hasegawai (Kamite, 2009) in Japan, neither of which were included in the present study. Genetic and developmental factors that affect body shape and hindwing length will be important subjects of future studies.

ACKNOWLEDGEMENTS. We thank H. Yoshitomi, Y. Kamite and J. Nakajima for information on hind-wing condition in some Japanese elmids, and H. Kadowaki, G. Yoshinari and J. Fujiwara for help with the sampling. This study was partly supported by a Grant-in-Aid for the Global COE Program (06) from the Ministry of Education, Culture, Sports, Science and Technology of Japan. S.D.S. was supported by a JSPS Postdoctoral Fellowship for Foreign Researchers from the Japan Society for the Promotion of Science.

\section{REFERENCES}

Brown H.P. 1987: Biology of riffle beetles. - Annu. Rev. Entomol. 32: 253-273.
Chenna R., Sugawara H., Koike T., Lopez R., Gibson T.J., HigGINS D.G. \& Thompson J.D. 2003: Multiple sequence alignment with the Clustal series of programs. - Nucl. Acids Res. 31: 497-500.

Clary D.O. \& Wolstenholme D.R. 1985: The mitochondrial DNA molecular of Drosophila yakuba: nucleotide sequence, gene organization, and genetic code. - J. Mol. Evol. 22: 252-271.

ElLiott J.M. 2008: The ecology of riffle beetles (Coleoptera: Elmidae). - Freshwater Rev. 1: 189-203.

Hayashi M. 2011: Aquatic Coleoptera of Shimane Prefecture. Spec. Bull. Hoshizaki Green Found. 1: 1-117.

Hayashi M. \& Sota T. 2008: Discrimination of two Japanese water pennies, Eubrianax granicollis Lewis and E. ramicornis Kiesenwetter (Coleoptera: Psephenidae), based on laboratory rearing and molecular taxonomy. - Entomol. Sci. 11: 349-357.

Hayashi M. \& Sota T. 2010: Identification of elmid larvae (Coleoptera: Elmidae) from Sanin District of Honshu, Japan, based on mitochondrial DNA sequences. - Entomol. Sci. 13: 417-424.

Hayashi M., Song S.D. \& Sota T. 2012: Molecular phylogeny and divergence time of the water penny genus Eubrianax (Coleoptera: Psephenidae) in Japan. - Entomol. Sci. 15: 314-323.

Huelsenbeck J.P. \& Ronquist F. 2001: MRBAYES: Bayesian inference of phylogenetic trees. - Bioinformatics 17: 754-755.

Hunt T., Bergsten J., Levkanicova Z., Papadopoulou A., St. John O., Wild R., Hammond P.M., Ahrens D., Balke M., Caterino M.S., Gómez-Zurita J., Ribera I., Barraclough T.G., Bocakova M., Bocak L. \& Vogler A.P. 2007: A comprehensive phylogeny of beetles reveals the evolutionary origins of a superradiation. - Science 318: 1913-1916.

IKEDA H., NishiKawa M. \& Sota T. 2012: Loss of flight promotes beetle diversification. - Nat. Comm. 3: 648

Inoue D. \& NakajIma J. 2009: Aquatic Insects of Fukuoka Prefecture. Gyobu, Fukuoka [in Japanese].

JENG M.L. \& YANG P.S. 1998: Taxonomic review of the genus Grouvellinus Champion (Coleoptera: Elmidae) from Taiwan and Japan. - Proc. Entomol. Soc. Wash. 100: 526-544.

Jobb G. von, Haeseler A. \& Strimmer K. 2004: Treefinder: a powerful graphical analysis environment for molecular phylogenetics. - BMC Evol. Biol. 4: 18.

KAMITE Y. 2009: A revision of the genus Heterlimnius Hinton (Coleoptera, Elmidae). — Jap. J. Syst. Entomol. 15: 199-226.

Kamite Y. 2011: A Taxonomic and Phylogenetic Study on the Genus Heterlimnius and Its Complex (Coleoptera, Elmidae). $\mathrm{PhD}$ thesis, The United Graduate School of Agricultural Sciences, Ehime University, Matsuyama.

Kamite Y. 2012: The genus Heterlimnius (Coleoptera, Elmidae) of Japan. - Sayabane (N.S.) 8: 22-26 [in Japanese].

Kamite Y., Ogata T. \& Satô M. 2006: A new species of the genus Zaitzeviaria (Coleoptera, Elmidae) from Tsushima Islands, Japan. - Jap. J. Syst. Entomol. 12: 149-153.

KawaI T. \& TANida K. 2005: Aquatic Insects of Japan: Mannual with Keys and Illustrations. Tokai University Press, Hadano, 1342 pp. [in Japanese].

Kim C.G., Zhou H.Z., Imura Y., Tominaga O., Su Z.H. \& Osawa S. 2000: Pattern of morphological diversification in the Leptocarabus ground beetles (Coleoptera, Carabidae) as deduced from mitochondrial ND5 gene and nuclear 28S rDNA sequences. - Mol. Biol. Evol. 17: 137-145.

Ogata T. \& Nakajima J. 2006: Elmid beetles of Fukuoka Prefecture, Northern Kyushu, Japan. - Bull. Hoshizaki Green Found. 9: 227-243 [in Japanese]. 
RofF D.A. 1986: The evolution of wing dimorphism in insects. - Evolution 40: 1009-1020.

RofF D.A. 1990: The evolution of flightlessness in insects. Ecol. Monog. 60: 389-421.

RofF D.A. 1994: The evolution of flightlessness: is history important? - Evol. Ecol. 8: 639-657.

RofF D.A. \& FAIRBAIRN D.J. 1991: Wing dimorphisms and the evolution of migratory polymorphisms among the Insecta. Am. Zool. 31: 243-251.

RofF D.A. \& FAIRBAIRn D.J. 2007: The evolution and genetics of migration in insects. - BioScience 57: 155-164.

SAtô M. 1985: Elmidae. In Ueno S., Kurosawa Y. \& Satô M. (eds): The Coleoptera of Japan in Color. Vol. 2. Hoikusha, Osaka, pp. 434-440, pls 79-80 [in Japanese].

SATô M. 1999: A new Stenelmis (Coleoptera, Elmidae) from Ryukyu Islands. - Entomol. Rev. Japan 54: 121-123.

SHEPARD W.D. 1998: Elmidae II. Description of Orientelmis gen. n. and new synonymy in Cleptelmis Sanderson (Coleoptera). In Jäch M.A. \& Ji L. (eds): Water Beetles of China II. Natural History Museum Vienna, Wien, pp. 289-295.

Simon C., Frati F., Beckenbach A., Crespi B., Liu H. \& Flook P. 1994: Evolution, weighting, and phylogenetic utility of mitochondrial gene sequences and a compilation of conserved polymerase chain reaction primers. - Ann. Entomol. Soc. Am. 87: 651-701.

SoutHWOOD T.R.E. 1962: Migration of terrestrial arthropods in relation to habitat. - Biol. Rev. 37: 171-214.

TANABE A.S. 2007: Kakusan: a computer program to automate the selection of a nucleotide substitution model and the configuration of a mixed model on multilocus data. - Mol. Ecol. Notes 7: 962-964.

WAGNer D.L. \& LiebHerR J.K. 1992: Flightlessness in insects. - Trends Ecol. Evol. 7: 216-220.

Yoshitomi H. \& NaKajIma J. 2007: A new species of the genus Sinonychus (Coleoptera, Elmidae) from Japan. - Elytra 35: 96-101.

Yoshitomi H. \& SAтô M. 2005: A revision of the Japanese species of the genus Dryopomorphus (Coleoptera, Elmidae). Elytra 33: 455-473.

Received December 19, 2012; revised and accepted April 23, 2013

APPENDIX ONLINE:

$<$ http://www.eje.cz/supplfiles/eje1844suppl.pdf $>$ 